\title{
TORUS INVARIANCE FOR THE CLIFFORD ALGEBRA. I
}

\author{
BY \\ MICHAEL C. REED
}

\begin{abstract}
A problem in Quantum Field Theory leads to the study of a representation of the torus, $T^{3}$, as automorphisms of the infinite dimensional Clifford algebra. It is shown that the irreducible product representations of the Clifford algebra fall into two categories: the discrete representations where the automorphisms are unitarily implementable, and all the others in which the automorphisms are not implementable and which cannot even appear as subrepresentations of larger representations in which the automorphisms are implementable.
\end{abstract}

I. Introduction. Let $X$ be a real infinite-dimensional Hilbert space, $C(X)$ the associated Clifford algebra, we denote the unique $C^{*}$-closure of $C(X)$ by $\mathscr{A}$. Let $\left\{x_{k}, y_{k} ; \boldsymbol{k}=\left(k_{1}, k_{2}, k_{3}\right) d, k_{i}\right.$ an arbitrary integer, $d>0$ fixed $\}$ be an orthonormal basis for $X$. Let $O(\alpha)$ denote the orthogonal transformation on $X$ which sends

$$
\begin{aligned}
& x_{k} \rightarrow x_{k} \cos (\boldsymbol{k} \cdot \boldsymbol{\alpha})-y_{k} \sin (\boldsymbol{k} \cdot \boldsymbol{\alpha}), \\
& y_{k} \rightarrow x_{\boldsymbol{k}} \sin (\boldsymbol{k} \cdot \boldsymbol{\alpha})+y_{k} \cos (\boldsymbol{k} \cdot \boldsymbol{\alpha}),
\end{aligned}
$$

where $\boldsymbol{\alpha}=\left(\alpha_{1}, \alpha_{2}, \alpha_{3}\right) \in \boldsymbol{R}^{3}$. The transformation $O(\alpha)$ induces an automorphism $\tau_{\alpha}$ of $C(X)$ and thus of $\mathscr{A}$. The map

$$
T^{3} \stackrel{\tau}{\longrightarrow} \text { Aut }(\mathscr{A})
$$

is a strongly continuous representation of the torus $T^{3}$ as automorphisms of $\mathscr{A}$. The problem is to classify those representations, $\pi(\cdot)$, of $\mathscr{A}$ on separable Hilbert spaces in which $\tau_{\alpha}$ is unitarily implementable, that is, in which there is a strongly continuous unitary representation, $U(\alpha)$, of $T^{3}$ on the separable Hilbert space such that $\pi\left(\tau_{\alpha}(A)\right)=U(\alpha) \pi(A) U(-\alpha)$. In this paper we begin a study of this problem by treating the most accessible class of representations, namely the irreducible tensor product representations. These representations fall into two classes: the discrete ones in which $\tau_{\alpha}$ is trivially unitarily implementable, and all the others in which $\tau_{\alpha}$ is not unitarily implementable and which cannot even appear as subrepresentations of larger representations in which $\tau_{\alpha}$ is unitarily implementable.

The reader is referred to the paper of Shale and Stinespring [6] for the construction and basic properties of the algebras $C(X)$ and $\mathscr{A}$. The fact that $\tau_{\alpha}$ is implementable in the discrete representations is already contained in their paper. For information about infinite tensor products of Hilbert spaces, the reader is referred to [4] or the brief summary in the appendix of [5].

Received by the editors April 6, 1970.

AMS 1970 subject classifications. Primary 46L05; Secondary 81A17, 81A20.

Key words and phrases. Clifford algebra, translation invariance, unitary implementability. Copyright (1) 1971, American Mathematical Society 
It is appropriate to briefly describe the origin of this problem in Quantum Field Theory. If we quantize in a three dimensional cube $B$, of side length $b$, the Fermion field

$$
\varphi(f)=b^{-3 / 2} \sum_{k=\left(k_{1}, k_{2}, k_{3}\right) 2 \pi / b}\left(f, e^{-i k \cdot x}\right)_{L^{2}(B)} a_{k}+\left(f, e^{i k \cdot x}\right)_{L^{2}(B)} a_{k}^{*}
$$

is an operator-valued distribution from $L^{2}(B)$ to the bounded operators on any separable Hilbert space, $\mathscr{H}$, on which the operators $\left\{a_{k}, a_{k}^{*}\right\}_{k}$ give a representation of the canonical anticommutation relations. That is, the operators $\left\{a_{k}, a_{k}^{*}\right\}_{k}$ are assumed to satisfy

$$
\left[a_{k}, a_{l}\right]_{+}=0=\left[a_{k}^{*}, a_{l}^{*}\right]_{+}, \quad\left[a_{k}, a_{l}^{*}\right]_{+}=\delta_{k l} I .
$$

If we identify opposite faces of the cube, then $B=T^{3}$, and the group of translations modulo the cube is also $T^{3}$. These translations induce a unitary action of $T^{3}$ on $L^{2}\left(T^{3}\right)$ by $f(x) \rightarrow f(x-\alpha)$ which in turn induces the action

$$
\varphi(f(x)) \rightarrow \varphi(f(x-\alpha))
$$

on the Fermion field. One is mainly interested in representations of the anticommutation relations in which there is a continuous unitary representation of the torus, $U(\alpha)$, such that

$$
\varphi(f(\boldsymbol{x}-\boldsymbol{\alpha}))=U(\boldsymbol{\alpha}) \varphi(f(\boldsymbol{x})) U(-\boldsymbol{\alpha})
$$

which requires $e^{i k \cdot \alpha} a_{k}=U(\alpha) a_{k} U(-\alpha)$ and $e^{-i k \cdot \alpha} a_{k}^{*}=U(\alpha) a_{k}^{*} U(-\alpha)$. Such a representation is called translation (modulo the cube) invariant. The smallest $C^{*}$-algebra containing $\left\{a_{k}, a_{k}^{*}\right\}_{k}$ gives a representation of $C(X)$, the correspondence being

$$
\pi\left(x_{k}\right)=(1 / \sqrt{ } 2)\left(a_{k}+a_{k}^{*}\right), \quad \pi\left(y_{k}\right)=(1 / i \sqrt{ } 2)\left(a_{k}-a_{k}^{*}\right) .
$$

Thus a representation of the canonical anticommutation relation will be translation invariant if and only if $\tau_{\alpha}$ is unitarily implementable in the corresponding representation of the Clifford algebra, $C(X)$, and its $C^{*}$-closure, $\mathscr{A}$.

II. Irreducible product representations. Let $\mathscr{K}$ be the lattice of points

$$
\left\{\left(k_{1}, k_{2}, k_{3}\right) d ; d>0, k_{i} \text { an integer }\right\}
$$

linearly ordered, starting with $(0,0,0)$, so that first the sphere about $(0,0,0)$ of radius $d$ is exhausted, then the sphere of radius $\sqrt{ } 2 d$ and so forth. $\boldsymbol{k}_{-}$will always indicate the predecessor of $\boldsymbol{k}, \boldsymbol{k}_{+}$the successor.

Let $C_{k}^{2}$ denote distinct copies of a two dimensional vector space over the complex numbers. We will denote by $H_{v}$ the closed separable subspace of $H=\bigotimes_{k} C_{k}^{2}$ generated by the $c_{0}$-vector

$$
v=\bigotimes_{\boldsymbol{k}}\left(\begin{array}{c}
\beta_{\boldsymbol{k}}^{1} \\
\beta_{\boldsymbol{k}}^{0}
\end{array}\right)
$$


where $\left|\beta_{k}^{1}\right|^{2}+\left|\beta_{k}^{0}\right|^{2}=1$. On each space $H_{v}$, the operators

$$
\begin{aligned}
& a_{p}=\left[\bigotimes_{k=0}^{p}\left(\begin{array}{rr}
-1 & 0 \\
0 & 1
\end{array}\right)\right] \otimes\left(\begin{array}{ll}
0 & 0 \\
1 & 0
\end{array}\right) \otimes\left[\bigotimes_{k=p_{+}}^{\infty} I_{k}\right], \\
& a_{p}^{*}=\left[\bigotimes_{k=0}^{p}\left(\begin{array}{rr}
-1 & 0 \\
0 & 1
\end{array}\right)\right] \otimes\left(\begin{array}{ll}
0 & 1 \\
0 & 0
\end{array}\right) \otimes\left[\bigotimes_{k=p_{+}}^{\infty} I_{k}\right]
\end{aligned}
$$

( $I_{k}$ denotes the identity operator on $C_{k}^{2}$ )

satisfy the canonical anticommutation relations. The family of operators $\left\{a_{p}, a_{p}^{*} ; p \leqq l\right\}$ on $\bigotimes_{p=0}^{l} C_{p}^{2}$ are the standard representation of the anticommutation relations [2] which is irreducible. Thus the operators $\left\{a_{p}, a_{p}^{*} ; \boldsymbol{p} \leqq l\right\}$ on $H_{v}$ generate a $C^{*}$-algebra which is the image of all the bounded operators on $\otimes_{0}^{l} C_{p}^{2}, \mathscr{B}\left(\otimes_{0}^{l} C_{p}^{2}\right)$, in the bounded operators on $H_{v}, \mathscr{B}\left(H_{v}\right)$, under the natural injection; we denote the image also by $\mathscr{B}\left(\bigotimes_{p=0}^{l} C_{p}^{2}\right)$. Since the $C^{*}$-algebra, $\mathscr{A}_{v}$, generated by the $a_{p}, a_{p}^{*}$ contains $\mathscr{B}\left(\bigotimes_{p=0}^{l} C_{p}^{2}\right)$ for each $l$, it equals the norm closure of $\cup_{l} \mathscr{B}\left(\bigotimes_{p=0}^{l} C_{p}^{2}\right)$. Although the algebras $\mathscr{A}_{v}$ for different $v$ are isomorphic we keep the index $v$ to indicate that $\mathscr{A}_{v}$ is the representation of $\mathscr{A}$ (the $C^{*}$-closure of the Clifford algebra) on $H_{v}$.

The algebras $\mathscr{A}_{v}$ and $\mathscr{A}_{w}$ are spatially isomorphic if and only if $v$ and $w$ are weakly equivalent [3], [4] which means that $\sum_{k}|1-|\left(v_{k}, w_{k}\right)||<\infty$ where $v$ $=\bigotimes_{k} v_{k}$ and $w=\bigotimes_{k} w_{k}$. Clearly, $\tau_{\alpha}$ is unitarily implementable in a representation of $\mathscr{A}$ if and only if it is implementable in all spatially isomorphic representations, so we need examine only one representation from each class.

A product representation is called discrete if it is spatially isomorphic to a representation $\mathscr{A}_{v}$ on $H_{v}$ whose generating vector

$$
v=\bigotimes_{k}\left(\begin{array}{l}
\beta_{k}^{1} \\
\beta_{k}^{0}
\end{array}\right)
$$

has either $\beta_{\boldsymbol{k}}^{0}=0$ or $\beta_{\boldsymbol{k}}^{1}=0$ for each $\boldsymbol{k} \in \mathscr{K}$. For such a representation define $\lambda: \mathscr{K} \rightarrow\{0,1\}$ by

$$
\begin{array}{rlrl}
\lambda(\boldsymbol{k}) & =0 & & \text { if } \beta_{\boldsymbol{k}}^{1}=0, \\
& =1 & \text { if } \beta_{\boldsymbol{k}}^{0}=0 .
\end{array}
$$

Then

$$
\begin{aligned}
U(\boldsymbol{\alpha}) & =\exp \left[-i \sum_{\boldsymbol{k}}(\boldsymbol{k} \cdot \boldsymbol{\alpha})\left(a_{\boldsymbol{k}}^{*} a_{\boldsymbol{k}}-\lambda(\boldsymbol{k}) I\right)\right] \\
& =\bigotimes_{\boldsymbol{k}}\left(\begin{array}{cc}
\exp [-i(\boldsymbol{k} \cdot \boldsymbol{\alpha})(1-\lambda(\boldsymbol{k}))] & 0 \\
0 & \exp [-i(\boldsymbol{k} \cdot \boldsymbol{\alpha}) \lambda(\boldsymbol{k})]
\end{array}\right)
\end{aligned}
$$

is a continuous unitary representation of $T^{3}$ which implements $\tau_{\alpha} . U(\alpha)$ makes sense and is a continuous unitary representation of $T^{3}$ on $H_{v}$ since on $c_{0}$-vectors, $w$, 
which differ from $v$ in only a finite number of components, all but a finite number of the operators in the infinite tensor product $(* *)$ act like the identity; thus $U(\boldsymbol{\alpha})$ is well defined on the set of finite linear combinations of such vectors which is dense in $H_{v}$. The fact that $U(\alpha)$ implements $\tau_{\alpha}$ is a calculation.

III. Implementability in irreducible product representations. The following theorem settles the question of implementability of $\tau_{\alpha}$ for the irreducible product representations:

THEOREM. Let $\mathscr{A}$ be the unique $C^{*}$-closure of the Clifford algebra of a real infinitedimensional Hilbert; $\tau_{\alpha}: T^{3} \rightarrow$ Aut $(\mathscr{A})$ the representation of the torus, $T^{3}$, as automorphisms of $\mathscr{A}$ described in $\S$ I. Let $\left\{\mathscr{A}_{v}, H_{v}\right\}$ be an irreducible product representation of $\mathscr{A}$. Then, if $\left\{\mathscr{A}_{v}, H_{v}\right\}$ is discrete, $\tau_{\alpha}$ is unitarily implementable. If $\left\{\mathscr{A}_{v}, H_{v}\right\}$ is not discrete, $\tau_{\alpha}$ is not implementable, and further, $\left\{\mathscr{A}_{v}, H_{v}\right\}$ cannot appear as a subrepresentation of a larger representation in which $\tau_{\alpha}$ is implementable.

We will use the following lemma which states that the contragredient action of $\tau_{\alpha}^{*}$ on any state $\omega \in \mathscr{A}^{*}$ must be continuous at the origin in $T^{3}$ if $\omega$ is a vector state of a representation of $\mathscr{A}$ in which $\tau_{\alpha}$ is implementable.

Lemma. Let $\pi(\cdot)$ be a representation of $\mathscr{A}$ on $\mathscr{H}$ in which $\tau_{\alpha}$ is implementable (by a continuous, unitary representation of $T^{3}$ ). Let $v \in \mathscr{H}$, then

$$
\sup _{A \in \mathscr{A},\|A\|=1}\left|\left(\pi\left(\tau_{\alpha}(A)\right) v, v\right)-(\pi(A) v, v)\right| \longrightarrow 0 \quad \text { as } \alpha \stackrel{T^{3}}{\longrightarrow} 0 .
$$

We omit the easy proof; an important converse statement, which says that if (3.1) holds for all $v \in \mathscr{H}$ then the representation can be imbedded in a larger one in which $\tau_{\alpha}$ is implementable, is due to H. Borchers [1].

We turn now to the proof of the theorem. For the discrete representations the implementing representation of $T^{3}$ is written down explicitly in §II. For the nondiscrete representations we show that (3.1) does not hold when

$$
v=\bigotimes_{k}\left(\begin{array}{l}
\beta_{k}^{1} \\
\beta_{k}^{0}
\end{array}\right)
$$

is a generating vector of $H_{v}$. We first suppose that the representation is nondiscrete and

$$
\min \left\{\beta_{\boldsymbol{k}}^{0}, \beta_{\boldsymbol{k}}^{1}\right\} \rightarrow 0 \text { as } \boldsymbol{k} \rightarrow \infty \text {. }
$$

Then there is an $\varepsilon>0$ and a subsequence $\left\{\boldsymbol{k}_{n}\right\}_{n=1}^{\infty}$ such that $\left|\beta_{\boldsymbol{k}_{n}}^{0}\right|\left|\beta_{\boldsymbol{k}_{n}}^{1}\right| \geqq \varepsilon^{2}$ for all $n$. Define

$$
\tilde{a}_{k}=\left[\prod_{p=0}^{k}\left(I-2 a_{p}^{*} a_{p}\right)\right] a_{k}=\left(\bigotimes_{p=0}^{k} I_{p}\right) \otimes\left(\begin{array}{ll}
0 & 0 \\
1 & 0
\end{array}\right) \otimes\left(\bigotimes_{p=k_{+}}^{\infty} I_{p}\right) .
$$

Then, $\tilde{a}_{k} \in \mathscr{A}_{v},\left\|\tilde{a}_{k}\right\|=1$ and

$$
\left|\left(\tau_{\alpha}\left(\tilde{a}_{k}\right) v, v\right)-\left(\tilde{a}_{k} v, v\right)\right|=\left|e^{i k \cdot \alpha}-1\right|\left|\beta_{k}^{0}\right|\left|\beta_{k}^{1}\right| .
$$


Thus, whatever $\delta>0$ is given we can find an $\boldsymbol{\alpha},|\boldsymbol{\alpha}|<\delta$ and an $n$ so that $\left|\left(\tau_{\alpha}\left(\tilde{a}_{k_{n}}\right) v, v\right)-\left(\tilde{a}_{k_{n}} v, v\right)\right| \geqq \varepsilon^{2}$ which shows that (3.1) does not hold.

It remains to consider the (more difficult) case where $\min \left\{\beta_{\boldsymbol{k}}^{0}, \beta_{\boldsymbol{k}}^{1}\right\} \rightarrow 0$. Let $\sigma: \mathscr{K} \rightarrow\{0,1\}$ be the function so that $\beta_{k}^{\sigma(k)} \rightarrow 0, \tilde{\sigma}(\cdot)$ the function which takes the value one when $\sigma$ is zero and vice versa. Then we must have $\sum_{k}\left|\beta_{k}^{\sigma(k)}\right|^{2}=\infty$. Otherwise, we let $w=\bigotimes_{k} w_{k}$ where $w_{k}=\left(\begin{array}{l}0 \\ 1\end{array}\right)$ if $\sigma(k)=1$ and $\left(\begin{array}{l}1 \\ 0\end{array}\right)$ if $\sigma(k)=1$. Then $v$ would be weakly equivalent to $w$ since

$$
\begin{aligned}
\sum_{k}|1-|\left(v_{k}, w_{k}\right)|| & =\sum_{k}|1-| \beta_{k}^{\tilde{\sigma}(k)}|| \\
& =\sum_{k}\left|1-\left(1-\left|\beta_{k}^{\sigma(k)}\right|^{2}\right)^{1 / 2}\right| \leqq c \sum_{k}\left|\beta_{k}^{\sigma(k)}\right|^{2}<\infty
\end{aligned}
$$

which contradicts the hypothesis that $v$ is nondiscrete; therefore $\sum_{k}\left|\beta_{k}^{\sigma(k)}\right|^{2}=\infty$. We may also assume that $0 \neq \beta_{k}^{\sigma(k)} \neq 1$ since there is another $c_{0}$-vector $u$ in $H_{v}$ with that property and we can replace $v$ by $u$.

Now, suppose $\delta>0$ is given. We will construct an operator $A_{N} \in \mathscr{A}_{v},\left\|A_{N}\right\|=1$, and an $\alpha$ with $\left|\alpha_{i}\right|<\delta$ so that $\left|\left(\tau_{\alpha}\left(A_{N}\right) v, v\right)-(A v, v)\right| \geqq \eta / 2>0$ where $\eta$ is independent of $\delta$ and $A_{N}$. Let $S_{i}=\{\boldsymbol{k} ; \boldsymbol{k} \in \mathscr{K}, \sigma(\boldsymbol{k})=i\}, \quad i=0,1$. Since $\sum\left|\beta_{\boldsymbol{k}}^{\sigma(k)}\right|^{2}=\infty$, $\prod_{k=0}^{p}\left|\beta_{k}^{\tilde{\sigma}(k)}\right|^{2} \rightarrow 0$ as $\boldsymbol{p} \rightarrow \infty$. Thus, either $\prod_{S_{0}}^{p}\left|\beta_{k}^{\tilde{\sigma}(k)}\right|^{2} \rightarrow 0$ or $\prod_{S_{1}}^{p}\left|\beta_{k}^{\tilde{\sigma}(k)}\right|^{2} \rightarrow 0$; we suppose the latter (the proof is not different in the other case); we may suppose further that $S_{1}$ is contained in one octant. Since $\left|\beta_{\boldsymbol{k}}^{\tilde{\sigma}(k)}\right| \rightarrow 1$ and $S_{1}$ is infinite we can choose finite, disjoint subsets $R_{1} \subset S_{1}$ and $R_{2} \subset S_{1}$ so that

(1) $\frac{1}{2} \leqq \prod_{R_{1}}\left|\beta_{\boldsymbol{k}}^{\tilde{\sigma}(k)}\right|^{2} \leqq \frac{3}{4}$.

(2) $\frac{1}{2} \leqq \prod_{R_{2}}\left|\beta_{k}^{\tilde{\sigma}(k)}\right|^{2} \leqq \frac{3}{4}$.

(3) For each $k \in R_{2}, \max _{i=1,2,3}\left|k_{i}\right| \geqq N+\sum_{k \in R_{1}}\left(\left|k_{1}\right|+\left|k_{2}\right|+\left|k_{3}\right|\right.$ ) (where $N$ is a positive integer).

Let

$$
w=\left(\bigotimes_{k \in R_{1}} v_{k}\right) \otimes\left(\bigotimes_{k \in R_{2}} v_{k}\right) \in h=\left(\bigotimes_{k \in R_{1}} C_{k}^{2}\right) \otimes\left(\bigotimes_{k \in R_{2}} C_{k}^{2}\right)
$$

Then $w$ can be written as a sum of eigenvectors (not necessarily normalized) of $\sum_{k \in R_{1} \cup R_{2}} a_{k}^{*} a_{k}$; let $\left\{f_{j}\right\}_{j=1}^{M_{1}}$ denote the eigenvectors of the form $u_{1} \otimes u_{2}$ where $u_{2}$ has eigenvalue zero and $u_{1}$ does not; let $\left\{g_{j}\right\}_{j=1}^{M_{2}}$ denote the eigenvectors of the form $u_{1} \otimes u_{2}$ where $u_{1}$ has eigenvalue zero and $u_{2}$ does not. Then

(1) $\left\{f_{j}\right\}_{j=1}^{M_{1}},\left\{g_{j}\right\}_{j=1}^{M_{2}}$ are all orthogonal vectors in $h$.

(2) $\frac{1}{8} \leqq \sum_{j=1}^{M_{1}}\left\|f_{j}\right\|^{2} \leqq \frac{1}{2}$ and $\frac{1}{8} \leqq \sum_{j=1}^{M_{2}}\left\|g_{j}\right\|^{2} \leqq \frac{1}{2}$.

(3) Each $f_{i}$ and $g_{j}$ is an eigenfunction of $\sum_{k \in R_{1} \cup R_{2}} k_{l} a_{k}^{*} a_{k}, l=1,2,3$, and for some $l$ the absolute value of the eigenvalue corresponding to $f_{i}$ plus $N$ is less than the absolute value of the eigenvalue corresponding to $g_{j}$.

We now define an operator $A_{N}$ on $h$ as follows. Suppose the element of the collection $\left\{f_{j}\right\}_{j=1}^{M_{1}},\left\{g_{j}\right\}_{j=1}^{M_{2}}$ with the largest norm is among the $f$ 's, call it $\hat{f}_{1}$. Then choose a sequence of $g$ 's, call them $\hat{g}_{1}, \ldots, \hat{g}_{r_{1}}$, which satisfy

$$
\frac{1}{8} \sum_{i=1}^{r_{1}}\left\|\hat{g}_{i}\right\|^{2} \leqq\left\|\hat{f}_{1}\right\|^{2} \leqq 8 \sum_{i=1}^{r_{1}}\left\|\hat{g}_{i}\right\|^{2}
$$


and define

$$
A_{N} \hat{f}_{1}=0, \quad A_{N} \hat{g}_{j}=\left(\frac{\left\|\hat{g}_{j}\right\|^{2}}{\left\|\hat{f}_{1}\right\|\left(\sum_{i}^{r_{1}}\left\|g_{j}\right\|^{2}\right)^{1 / 2}}\right) \hat{f}_{1}
$$

and extend $A_{N}$ by linearity to the subspace $\mathscr{M}_{1}$ spanned by $\hat{f}_{1}$ and $\left\{\hat{g}_{i}\right\}_{i=1}^{r_{1}}$. Then $\left\|A_{N}\right\| \leqq 1$ on $\mathscr{M}_{1}$ and

$$
\left(A_{N}\left(\hat{f}_{1}+\sum^{r_{1}} \hat{g}_{i}\right),\left(\hat{f}_{1}+\sum^{r_{1}} \hat{g}_{i}\right)\right)=\sum_{j=1}^{r_{1}} t_{1 j}^{2} \geqq \frac{1}{4}\left\|\hat{f}_{1}\right\|^{2} .
$$

We now return to the remaining members of the collection $\left\{f_{j}\right\}_{j=1}^{M_{1}},\left\{g_{j}\right\}_{j=1}^{M_{2}}$, find the element with largest norm and proceed to define $A_{N}$ on a new subspace $\mathscr{M}_{2}$ orthogonal to $\mathscr{M}_{1}$ and so forth. When either the sum of the squares of the norms of the elements left in $\left\{f_{j}\right\}_{j=1}^{M_{1}}$ or in $\left\{g_{j}\right\}_{j=1}^{M_{2}}$ is $<\frac{1}{16}$, we stop and define $A$ to be the zero operator on the rest of $h$ (requiring that the sums of squares of the norms be $\geqq \frac{1}{16}$ in both collections insures that an inequality like (3.2) will be true). On $h$, $\left\|A_{N}\right\| \leqq 1$ and

$$
\left(A_{N}\left(\sum_{j=1}^{M_{1}} f_{j}+\sum_{j=1}^{M_{2}} g_{j}\right),\left(\sum_{j=1}^{M_{1}} f_{j}+\sum_{j=1}^{M_{2}} g_{j}\right)\right)=\sum_{l=1}^{L} \sum_{j=1}^{r_{l}} t_{l j}^{2} \geqq\left(\frac{1}{4}\right)\left(\frac{1}{16}\right)=2^{-6} .
$$

Since $A_{N}$ is a bounded operator on $h=\left(\bigotimes_{R_{1}} C_{k}^{2}\right) \otimes\left(\bigotimes_{R_{2}} C_{k}^{2}\right), A_{N} \in \mathscr{A}_{v}$, and $\tau_{\alpha}\left(A_{N}\right)=U_{0}(\alpha) A_{N} U_{0}(-\alpha)$ where $U_{0}(\alpha)=\exp \left[i \sum_{R_{1} \cup R_{2}}(\alpha \cdot k) a_{k}^{*} a_{k}\right]$, since $R_{1}$ and $R_{2}$ are finite sets. Thus

$$
\begin{aligned}
\left(\tau_{\alpha}\left(A_{N}\right) v, v\right)_{H_{V}} & =\left(U_{0}(\alpha) A_{N} U_{0}(-\alpha) v, v\right)_{H_{v}} \\
& =\left(A_{N} U_{0}(-\alpha) w, U_{0}(-\alpha) w\right)_{n} \\
& =\sum_{n=1}^{n_{0}} e^{i \alpha \cdot \varphi(n)} t_{n}^{2}
\end{aligned}
$$

where $\sum_{n=1}^{n_{0}} t_{n}^{2}=\eta \geqq 2^{-6}$ and $\varphi(n)=\left(\varphi_{1}(n), \varphi_{2}(n), \varphi_{3}(n)\right)$ has the property that, for each $n$, there is some $i$ for which $\left|\varphi_{i}(n)\right| \geqq N$ (this follows from (3)) above and the fact that $A$ takes the $f$ 's into the $g$ 's and vice versa). Suppose $\mathscr{R} e\left(\tau_{\alpha}(A) v, v\right) \geqq \eta / 2$ for all $\alpha$ inside a cube centered at $(0,0,0)$ of side length $2 \delta$. Then

$$
\mathscr{R} e \int_{0}^{\delta} \int_{0}^{\delta} \int_{0}^{\delta}\left(\tau_{\alpha}\left(A_{N}\right) v, v\right) d \alpha=\int_{0}^{\delta} \int_{0}^{\delta} \int_{0}^{\delta} \mathscr{R} e\left(\tau_{\alpha}\left(A_{N}\right) v, v\right) d \alpha \geqq \frac{\delta^{3} \eta}{2}
$$

but

$$
\mathscr{R} e \int_{0}^{\delta} \int_{0}^{\delta} \int_{0}^{\delta}\left(\tau_{\alpha}\left(A_{N}\right) v, v\right) d \alpha=\mathscr{R} e\left\{\sum_{n=1}^{n_{0}}\left(\frac{1}{i \varphi_{1}(n) \varphi_{2}(n) \varphi_{3}(n)}\right)\left(e^{i \delta \cdot \varphi(n)}-1\right) t_{n}^{2}\right\} \leqq \frac{2 \eta}{N}
$$

which is a contradiction if $N$ is large enough. Therefore, if $N$ is large enough we have $\mathscr{R} e\left\{\left(\tau_{\alpha_{0}}\left(A_{N}\right) v, v\right)\right\} \leqq \eta / 2$, for some $\alpha_{0}=\left(\alpha_{1}^{0}, \alpha_{2}^{0}, \alpha_{3}^{0}\right)$ with $\left|\alpha_{i}^{0}\right| \leqq \delta$. That is

$$
\left|\left(\tau_{\alpha_{0}}\left(A_{N}\right) v, v\right)-\left(A_{N} v, v\right)\right| \geqq \eta / 2 .
$$


Since for any $\delta>0$ we can find an $N$, a corresponding $A_{N}$, and an $\alpha_{0}$ so that the inequality (3.3) holds,

$$
\sup _{A \in \mathscr{A}_{v},\|A\| \leqq 1}\left|\left(\tau_{\alpha}(A) v, v\right)-(A v, v)\right| \nrightarrow 0 \quad \text { as } \alpha \stackrel{T^{3}}{\longrightarrow} 0 .
$$

This completes the proof of the theorem.

ACKNOWLEDGEMENTS. The question of torus invariance was suggested to the author by A. S. Wightman. The author had several helpful discussions with Hale Trotter and Gianfausto Dell'Antonio.

\section{BIBLIOGRAPHY}

1. H. Borchers, On the implementability of automorphism groups, Comm. Math. Phys. 14 (1969), 305.

2. P. Jordan and E. Wigner, Über das Paulische Äquivalenzverbot, Z. Phys. 47 (1928), 631.

3. J. R. Klauder, J. McKenna and E. J. Woods, Direct-product representations of the canonical commutation relations, J. Mathematical Phys. 7 (1966), 822-828. MR 33 \#7004.

4. J. von Neumann, On infinite direct products, Compositio Math. 6 (1938), 1-77.

5. M. Reed, On self-adjointness in infinite tensor product spaces, J. Functional Anal. 5 (1970), 94.

6. D. Shale and W. F. Stinespring, States of the Clifford algebra, Ann. of Math. (2) 80 (1964), 365-381. MR 29 \#3160.

Princeton University,

Princeton, New Jersey 08540 